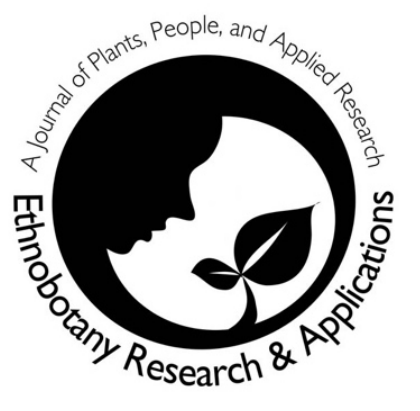

\title{
Ethnobotanical profile of some useful plants and fungi of district Dir Upper, Tehsil Darora, Khyber Pakhtunkhwa, Pakistan
}

Murad Muhammad, Lal Badshah, Adnan Ali Shah, Abdullah, Muhammad Ali Shah, Rainer W. Bussmann and Abdul Basit

\section{Databases and Inventories}

\begin{abstract}
Background: The present study was conducted to explore the ethnobotanical and ethnomedicinal uses of plants in 12 remote villages of Tehsil Darora, district Dir Upper, Pakistan.
\end{abstract}

Methods: Among the residents both, men, and women (some traditional healers like Hakeen and Pansires) were interviewed about the useful plants existing in the area using comprehensive questionnaires.

Results: A total of 91 plant species belonging to 37 families were recorded in the four seasons (spring, summer, autumn, winter) in 2019-2020. These included 86 angiosperms (94\%) 2 gymnosperms (2\%) 2 fungi (2\%) and 2 pteridophytes (2\%). Asteraceae was the dominant family having 9 species, followed by Lamiaceae (8 species), 6 species of Amaranthaceae, and Cucurbitaceae, Fabaceae (5 species), Euphorbiaceae, and Rosaceae, each with 4 species.

Conclusions: The documented plants were used by the local communities medicinally and for other purposes such as fuel, vegetables, fodder, shelter, and timber wood, etc.

Keywords: Ethnobotanical plants, Fungi, Tehsil Darora, District Dir Upper, Pakistan

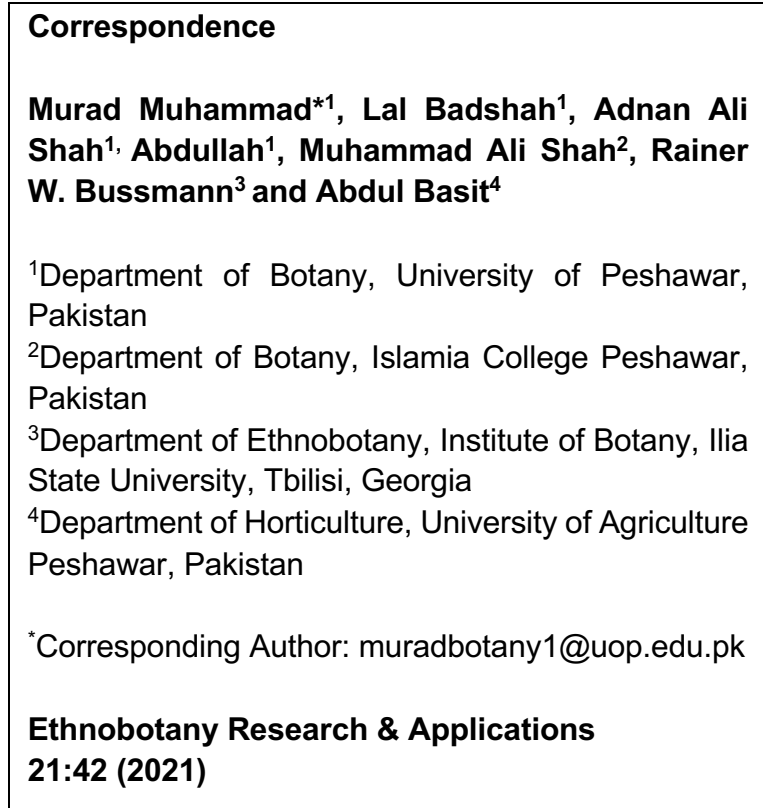

\section{Background}

Plants have been used as food, medicines and other purposes all though human history (Punchay et al. 2020; Balász et al. 2020). Ethnobotany is an extensive field researching human-plant interactions (Latimer 2020; Aloi 2018). Ethnobotanical studies point out locally important plant species and might provide leads for the discovery of crude and modern-day drugs (Elachouri et al. 2021). In Pakistan ethnomedicinal studies are quite new, about 6000 plant species have been reported from Pakistan (Abbas et al. 2017; Majid et al. 2019). Of the approximately 414000 flowering plants globally, 40000 have been reported for a variety of uses. According to World Health Organization (WHO), $80 \%$ of the global 
population uses plants for their primary health care (Mustafa et al. 2017). According to a survey in the early 1960 s approximately $76 \%$ of the population of Pakistan was directly linked to plant use, and plants were considered a big and effective source for medicines (Jan et al. 2019; Witt et al. 2018). The residents of the rural areas often have excellent knowledge about the application and utilization of plant species (Sutrisno et al. 2020; Dapar et al. 2020). To preserve the traditional knowledge and contribute to drug prospecting, ethnobotanical studies have been conducted in different regions of this country (Pandey \& Tripathi 2017).

The northern area of Pakistan has a rich history of folk knowledge regarding plants and animal resources (Abbas et al. 2020). Dir Upper in Khyber Pakhtunkhwa, Pakistan is a remote area since the day of independence where local communities always relied on medicinal plants for numerous diseases and have generally great dependency on plant products. During this survey, knowledge was obtained from the residents about the uses of different plants and fungal species which are used against different human and animal diseases. In addition medicinal uses of plant species, some plants were also used for various purposes such as a cosmetics agent, toothbrush, shelter, fuel, fodder, hedges, and furniture, etc. The current study was the first-ever systematic approach to document the uses of plants and some fungal species in the area. We intended to 1. Document plant uses in the area, 2. Gather data as baseline for better management practices, 3. The preservation of folk traditional knowledge and to make the data publicly available, and 4 . Alert and motivate the concerned population against the overexploitation of the unique medicinal flora and reckless clearance of forests and vegetation of the area. We hypothesized that due to its remoteness plant and animal use in the area would be different to surrounding areas and the wider region.

\section{Materials and Methods \\ Study Area}

The research was carried out during 2018-2020 in Tehsil Darora, District Dir Upper which covers about 3,699 $\mathrm{km}^{2}$ and comprises 7 tehsils divided into 28 union councils. According to the census 2017, its population was 946,421 (Elahi \& Rahman 2021). It borders District Chitral and Afghanistan on North and Northwest and District Swat at the east, and on the south joined the District Lower Dir (Figure.1).

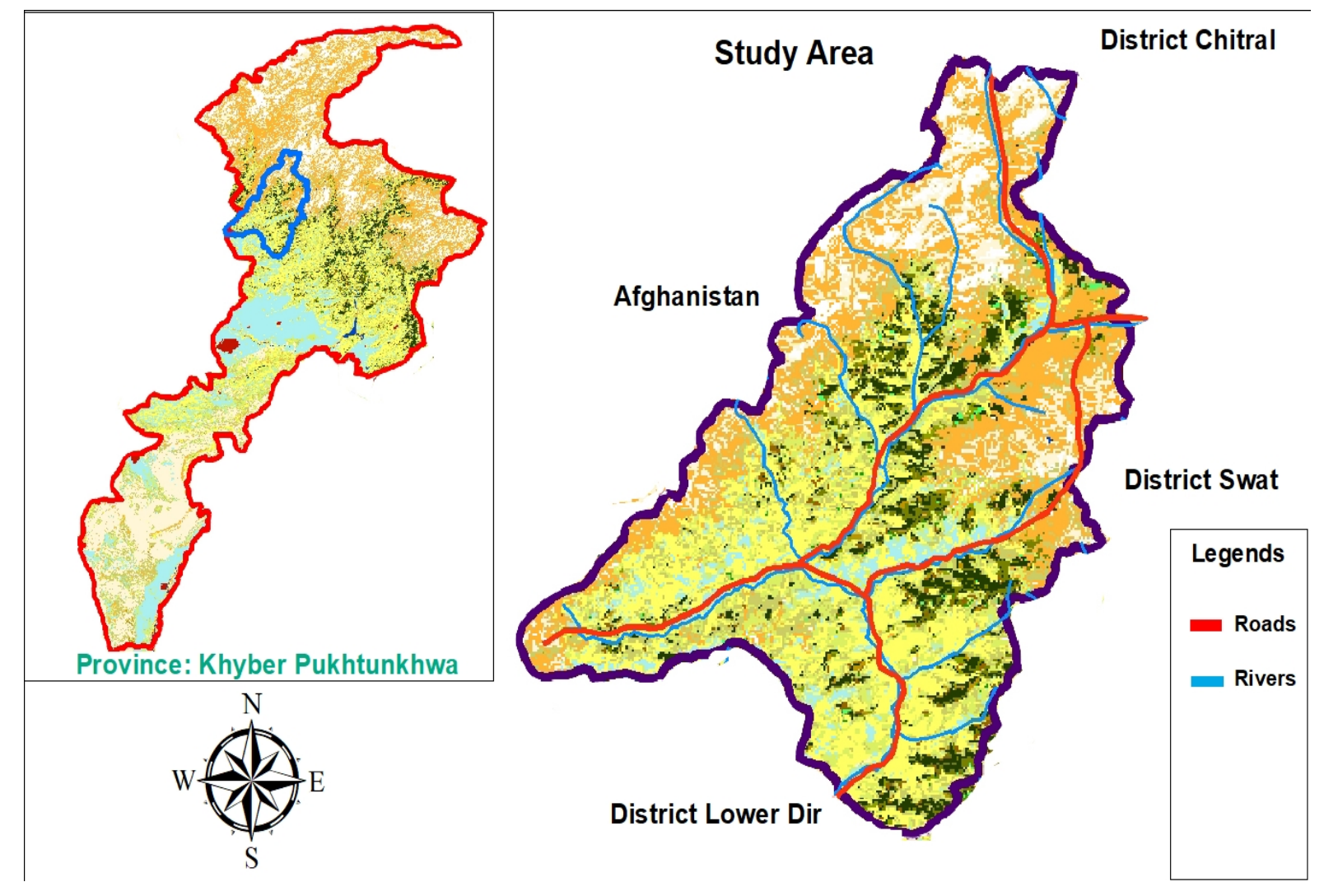

Figure 1. Study area map 
At the time of independence of Pakistan, Dir was a separate princely state under the aegis of Nawab shah Jehan Khan. In 1969 it was merged with Pakistan and was declared a district in 1970. The headquarter of District Dir is Chakdara. District Dir is a predominantly rural and hilly area situated in the northern part of Pakistan, divided into two subdivisions i.e., Dir Upper and Dir Lower. The research area tehsil Darora and their associated villages are situated in district Dir Upper. District Dir upper lies between $35^{\circ} 12^{\prime} 24^{\prime \prime} \mathrm{N}$ latitude and $71^{\circ} 52^{\prime} 36$ "E longitude, at an elevation of 1300-1500 m.a.s.l. It is administratively subdivided into seven tehsils which contain a total of 28 Union Councils. Tehsil Darora is situated in the south of Dir town (headquarter of Dir Upper). The research area, tehsil Darora is $23 \mathrm{~km}$ away (toward the south) from the headquarter of Upper Dir (the town of Dir). Climatically the research area falls in a humid subtropical climate zone with four distinct seasons (winter, spring, summer and, autumn). The winter season is harsh and long. Snowfall frequently happens in mid of the winter season (December and January) at high altitudes sites of the research area. The research covered 5 union councils, where the local inhabitants are Sultan Khel, Painda Khel, and Gujar with Pashto as native language. The residents of the area earn their livelihood from agriculture, selling bovine products, trading forest resources, and cattle rearing. Agriculture is the main source of income.

\section{Data collection}

Ethnobotanical data was collected on 16 study trips to representative areas, and specimens were collected in four different seasons. i.e., spring, summer, autumn, and winter. Information regarding ethnobotanical, ethnomycological and ethnozoological uses was collected using a detailed questionnaire (See appendix). Residents of different ages, and gender were interviewed after obtaining their prior informed consent. Informants were selected based on folk knowledge, locality, occupation, and age. Preference was given to the old and native peoples. Most of the interviewed informants were aged from 48 to 68 . Some female residents were also included in the survey knowledge, although cultural rules made interviewing women difficult. The majority of the informants were rural, by occupation they were farmers and plant traders. Some urban residents were also interviewed, and the majority were teachers and shopkeepers. All the included informants had great experience and knowledge about the medicinal plants. Ethnobotanical and ethnomedicinal data, the local name of the plants, traditional usage of plants, and other basic suggestions and recommendations were noted. Few short visits were also conducted to the local markets to verify and elaborate the collected information regarding medicinal plants (Figure.2). The Code of Ethics of the International Society of Ethnobiology was followed (Shinwari, 2010). The local language (Pashto) was used during the survey for the sake of ease and accuracy. The participants did not want demographic details to be disclosed.

Specimens of all plants were collected and identified by expert plant taxonomists at the Department of Botany, University of Peshawar, and Islamia College University Peshawar. For confirmation and authenticity of scientific names the Flora of Pakistan was accessed. The taxonomy was updated to APG using Tropicos.org. The specimens were deposited in the herbarium Department of Botany, University of Peshawar, Pakistan for future reference. The plants along with their scientific name, vernacular name, part used, and local uses have been listed in Table 1.

\section{Results}

\section{Ethnobotanical diversity}

Total of 92 species belonging to 37 families in which 2 species of pteridophytes (2\%) 2 fungal species $(2 \%)$ and 2 gymnosperms (2\%) were recorded. Asteraceae was the dominant family having 9 species, followed by Lamiaceae (8 species), Cucurbitaceae and Amaranthaceae (6 species), Fabaceae (5 species), Euphorbiaceae, and Rosaceae (4 species), Apiaceae (3 species) (Table 2). We found 61 herbs, 10 shrubs, 18 trees, and two fungi and their part used were enlisted, as shown in Figure 3 and 4.

\section{Disease Categories}

Out of all medicinal plants, the highest number (13) of plant species were used for gastrointestinal tract diseases (GIT) (30\%) followed by respiratory diseases (14\%). Some plant species were used for multiple ailments (e.g. Papaveraceae and Pinaceae). Some species were used against diabetes, fever, skin problems, renal problems, for wound healing, as body energizers, and anticonstipation (Figure 5). Earlier ethnobotanical research (Chetry et al. 2018; Umair et al. 2017; Santos et al. 2019) conducted in diverse areas also described the common usage of medicinal plants against gastrointestinal diseases. The reported plant species used against various diseases should be further appraised and examined through therapeutic and biological properties. 
Table 1. Collected plants in the local area and their ethnobotanical uses.

\begin{tabular}{|c|c|c|c|c|}
\hline $\begin{array}{l}\text { Family } \\
\text { Scientific name }\end{array}$ & Local name (Pashto) & Part used & Life form & Indigenous knowledge \\
\hline \multicolumn{5}{|l|}{ Adiantaceae } \\
\hline Adiantum capillus-veneris L. & Sumbal & $\begin{array}{l}\text { Shoots and } \\
\text { leaves }\end{array}$ & Herb & $\begin{array}{l}\text { Shoots and leaves are effective against coughs, whooping cough, } \\
\text { and other bronchitis infections. }\end{array}$ \\
\hline \multicolumn{5}{|r|}{ (4) } \\
\hline Agaricus campestris $\mathrm{L}$. & Khareray & Whole plant & Mushroom & Used as a nutritional food and quick source of energy. \\
\hline \multicolumn{5}{|l|}{ Alismataceae } \\
\hline Alisma plantago-aquatica L. & Ghawa jabai & Leaves & Herb & $\begin{array}{l}\text { Leaves are used for diabetes, also used for digestive and renal } \\
\text { problems. }\end{array}$ \\
\hline \multicolumn{5}{|l|}{ Amaranthaceae } \\
\hline Amaranthus viridis L. & Churlyai & Leaves & Herb & Cooked leaves are used as a vegetable. \\
\hline Chenopodium album L. & Churlayai & $\begin{array}{l}\text { Leaves and } \\
\text { shoots }\end{array}$ & Herb & $\begin{array}{l}\text { Leaves and shoots used as anthelmintic agent, fodder, common } \\
\text { source of vegetables. }\end{array}$ \\
\hline $\begin{array}{l}\text { Chenopodium ambrosioides (L.) Mosyakin \& } \\
\text { Clemants }\end{array}$ & Sakhaboty & $\begin{array}{l}\text { Leaves and } \\
\text { shoots }\end{array}$ & Herb & $\begin{array}{l}\text { Extracted juice from leaves and stems is used against common } \\
\text { and malarial fever, also used against abdominal heat. }\end{array}$ \\
\hline $\begin{array}{l}\text { Chenopodium botrys (L.) Mosyakin \& } \\
\text { Clemants }\end{array}$ & Kharawa & Seeds & Herb & $\begin{array}{l}\text { Seeds are used against fever especially malaria, for skin } \\
\text { problems, stomach-ache, and as blood thinner. }\end{array}$ \\
\hline $\begin{array}{l}\text { Chenopodium murale (L.) S. Fuentes, Uotila } \\
\text { \& Borsch }\end{array}$ & Churlayai & $\begin{array}{l}\text { Leaves and } \\
\text { shoots }\end{array}$ & Herb & $\begin{array}{l}\text { Leaves and shoots are used against abdominal problems and as } \\
\text { vegetables. }\end{array}$ \\
\hline Spinacia oleracea L. & Palak & $\begin{array}{l}\text { Leaves and } \\
\text { shoots }\end{array}$ & Herb & $\begin{array}{l}\text { Leaves and shoots are used as vegetables and to strengthen } \\
\text { bones. }\end{array}$ \\
\hline \multicolumn{5}{|l|}{ Amaryllidaceae } \\
\hline Allium cepa L. & Piaz & $\begin{array}{l}\text { Bulbs and } \\
\text { leaves }\end{array}$ & Herb & $\begin{array}{l}\text { Leaves are diuretic while bulbs are stimulant and decrease the } \\
\text { intensity of bites (dogs, insects) }\end{array}$ \\
\hline Allium sativum $\mathrm{L}$. & Ooga & $\begin{array}{l}\text { Bulb and } \\
\text { leaves }\end{array}$ & Herb & $\begin{array}{l}\text { Used as condiment, and expectorant, normalize lower blood } \\
\text { pressure. }\end{array}$ \\
\hline \multicolumn{5}{|l|}{ Apiaceae } \\
\hline Ammi visnaga Mill. & Sperkai & Whole plant & Herb & $\begin{array}{l}\text { Used against sharp fever, anti-asthmatic properties, also uses } \\
\text { against internal wounds, abdominal pain (mixed with jaggery and } \\
\text { boiled), coldness (for both human and livestock) }\end{array}$ \\
\hline Coriandrum sativum $\mathrm{L}$. & Dhanya & $\begin{array}{l}\text { Seeds and } \\
\text { leaves }\end{array}$ & Herb & $\begin{array}{l}\text { Seeds and leaves are widely used as condiments, aromatic and } \\
\text { flavoring agents, as a stimulating agent. }\end{array}$ \\
\hline Foeniculum vulgare Mill. & Kaaga & Seeds & Herb & $\begin{array}{l}\text { Seeds are used against any digestive and gastric problems, as an } \\
\text { appetizer, criminative }\end{array}$ \\
\hline \multicolumn{5}{|r|}{ 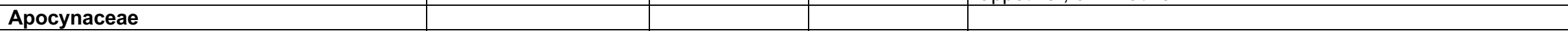 } \\
\hline Calotropis procera (Aiton) W.T. (Aiton) & Spalmai & Latex & Shrub & Diuretic, for muscular pain, against stomach-ache \\
\hline Periploca aphylla Decne. & ---------- & Leaves & Shrub & Leaves are used to cure gum diseases \\
\hline
\end{tabular}




\begin{tabular}{|c|c|c|c|c|}
\hline \multicolumn{5}{|l|}{ Asclepiadaceae } \\
\hline Artemisia vulgaris $\mathrm{L}$. & Tarkha & $\begin{array}{l}\text { Leaves and } \\
\text { young shoots }\end{array}$ & Herbs & Used against abdominal worms, anti-malarial \\
\hline \multicolumn{5}{|l|}{ Asphodelaceae } \\
\hline Asphodelus tenuifolius Cav. & -------- & Leaves & Herb & Leaves are used as condiment. \\
\hline \multicolumn{5}{|l|}{ Asteraceae } \\
\hline Artemisia scoparia Waldst. \& Kit. & Jawakay & $\begin{array}{l}\text { Stem and } \\
\text { leaves }\end{array}$ & Herb & Stem along with leaves used as broom, also used as fuel. \\
\hline Carthamus lanatus L. & Ghana & Whole plants & Herb & Used for living fences. \\
\hline Carthamus oxyacantha M. Bieb. & Ghana & Leaves & Herb & Leaves utilized as bandages for external wounds. \\
\hline Cichorium intybus L. & ----- & $\begin{array}{l}\text { Leaves and } \\
\text { flower }\end{array}$ & Herb & $\begin{array}{l}\text { Leaves as a vegetable and flowers/roots are used as coffee } \\
\text { replacement. }\end{array}$ \\
\hline Conyza canadensis (L.) Cronquist & Daniabotay & $\begin{array}{l}\text { Seeds and } \\
\text { leaves }\end{array}$ & Herb & $\begin{array}{l}\text { Leaves are cooked as vegetable, seeds cooked in combination } \\
\text { with rice. }\end{array}$ \\
\hline Helianthus annuus $\mathrm{L}$. & Namar parast & $\begin{array}{l}\text { Oil and seed } \\
\text { yields }\end{array}$ & Herb & $\begin{array}{l}\text { Oil used for cooking, seeds for anti-constipation and diuretic } \\
\text { properties. }\end{array}$ \\
\hline Sonchus asper (L) Hill. & Shawda boaty & $\begin{array}{l}\text { Ash and } \\
\text { leaves }\end{array}$ & Herb & Ash reduces bodypain, leaves are used as fodder for cattle. \\
\hline $\begin{array}{l}\text { Taraxacum officinale } \\
\text { (L.) Weber ex F.H. Wigg. }\end{array}$ & Ziar guly & Whole plant & Herb & $\begin{array}{l}\text { The boiled plant is used as a body regulating and stimulating } \\
\text { agent. }\end{array}$ \\
\hline Xanthium strumarium L. & Geshkay & $\begin{array}{l}\text { Leaves and } \\
\text { stem }\end{array}$ & Herb & Leaves are used against respiratory disorders and as antipyretic. \\
\hline \multicolumn{5}{|l|}{ Berberidaceae } \\
\hline Berberis lycium Royle. & Kwaray & $\begin{array}{l}\text { Fruits, roots, } \\
\text { and shoots }\end{array}$ & Shrub & $\begin{array}{l}\text { Fruits are used as a blood purifier, bark and root powder for } \\
\text { throat, and stomach ulcer /infection, fever. }\end{array}$ \\
\hline \multicolumn{5}{|r|}{ (1) } \\
\hline Brassica campestris L & Sharsham & Whole plant & Herb & $\begin{array}{l}\text { Fodder for cattle. Seed oils can be used for body massage, anti- } \\
\text { dandruff, and ointment, leaves, and flowers used as vegetable. }\end{array}$ \\
\hline Brassica oleracea L. & Zangali Falak & Whole plant & Herb & Vegetable and for salad \\
\hline Eruca sativa (L.) Cav. & Pana saag & $\begin{array}{l}\text { Barks and } \\
\text { leaves }\end{array}$ & Herb & $\begin{array}{l}\text { A rich source of nutrients (vegetable), boosting. } \\
\text { immune response. }\end{array}$ \\
\hline Lepidium sativum $\mathrm{L}$. & Gul saag & Whole plant & Herb & As a tonic, and anti-gastric \\
\hline Nasturtium officinale W.T. Aiton & Talmera & $\begin{array}{l}\text { Shoots and } \\
\text { leaves }\end{array}$ & Herb & $\begin{array}{l}\text { Shoots and leaves are used against tetanus and as common } \\
\text { vegetables. }\end{array}$ \\
\hline \multicolumn{5}{|l|}{ Cannabaceae } \\
\hline Cannabis sativa $\mathrm{L}$. & Kabaly bhang & $\begin{array}{l}\text { Seeds and } \\
\text { shoots }\end{array}$ & Herb & Used against insomnia, depression, and loss of appetite. \\
\hline \multicolumn{5}{|l|}{ Cucurbitaceae } \\
\hline Citrullus colocynthis (L.) Schrad. & Anda botay & Fruits & Herb & Used as a digestive agent, for digestive disorders. \\
\hline Cucumis melo L. & Andawna & Fruits & Herb & Fruits are edible, also used against water and blood deficiency. \\
\hline
\end{tabular}




\begin{tabular}{|c|c|c|c|c|}
\hline Cucumis sativus $\mathrm{L}$. & Baadrang & Fruits & Herb & $\begin{array}{l}\text { Uses in salad as a digestive agent, also used against extensive } \\
\text { thirst. }\end{array}$ \\
\hline Cucurbita maxima Duchesne & Khug kaddu & Fruit & Herb & $\begin{array}{l}\text { Used against stomach burns and joint inflammation, nervous } \\
\text { disorders, as common vegetable. }\end{array}$ \\
\hline Cucurbita pepo L. & Dobrr kadu & Fruits & Herb & $\begin{array}{l}\text { Fruits are used to treat general debility and sugar, used widely as } \\
\text { vegetable. }\end{array}$ \\
\hline Luffa acutangula (L.) Roxb. & Toorai & Fruits & Herb & Used as a vegetable and digestive tonic. \\
\hline \multicolumn{5}{|l|}{ Cupressaceae } \\
\hline Cupressus sempervirens L. & Sarwah & Whole plant & Tree & $\begin{array}{l}\text { Seeds are anthelmintic, widely used for ornamental purposes in } \\
\text { gardens, stem used as fuelwood. }\end{array}$ \\
\hline \multicolumn{5}{|r|}{ 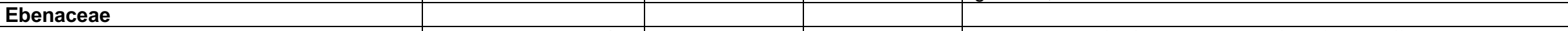 } \\
\hline Diospyros lotus L. & $\begin{array}{l}\text { Toormanzay amluk/ } \\
\text { farsimnn }\end{array}$ & $\begin{array}{l}\text { Fruits and } \\
\text { stem }\end{array}$ & Tree & $\begin{array}{l}\text { Wood is used for furniture and as fuel, fruit is effective against } \\
\text { flatulence. }\end{array}$ \\
\hline \multicolumn{5}{|l|}{ Equisetaceae } \\
\hline Equisetum arvense L. & Bandakay & Whole plant & Herb & $\begin{array}{l}\text { Used against small intestinal and urinary bladder inflammation and } \\
\text { kidney stones, fever. }\end{array}$ \\
\hline \multicolumn{5}{|l|}{ Euphorbiaceae } \\
\hline Euphorbia helioscopia L. & Mandanu & Milky sap & Herb & Latex is used to treat burns and skin eruptions. \\
\hline Euphorbia hirta L. & Shawda botay & Latex, shoot & Herb & Latex is used against skin problems, shoot as a fodder. \\
\hline Euphorbia prostrata Aiton. & Shawda botay & Shoots & Herb & Shoots are used against itching \\
\hline Mallotus philippensis (Lam.) Muell- Arg. & Dana botay & Fruit & Shrub & Fruits are anti-diarrheal, also used for other abdominal disorders. \\
\hline \multicolumn{5}{|l|}{ Fabaceae } \\
\hline Acacia arabica (Lam.) Willd. & Kikaar & $\begin{array}{l}\text { Leaves, Stem, } \\
\text { and seeds }\end{array}$ & Tree & $\begin{array}{l}\text { Boiled leaves are used against cough, seeds for dysentery, wood } \\
\text { used as fuel. }\end{array}$ \\
\hline Acacia nilotica (L.) Willd. ex Del. & Paloosa & $\begin{array}{l}\text { Gum, leaves, } \\
\text { bark }\end{array}$ & Tree & $\begin{array}{l}\text { Bark and branches used as toothbrush and fuelwood, leaves as } \\
\text { fodder, for gums, joints pain, against stomach ulcers. }\end{array}$ \\
\hline Dalbergia sissoo Roxb. & Shawa & Stem & Tree & $\begin{array}{l}\text { Wood is used for furniture (best source for cash income), and as } \\
\text { fuel }\end{array}$ \\
\hline Senegalia modesta (Wall.) P.J.H. Hurter & Watany / Kikaar & $\begin{array}{l}\text { Gum, leaves, } \\
\text { bark }\end{array}$ & Tree & Leaves are used for cough, intestinal infections. \\
\hline Trifolium repens $\mathrm{L}$. & Shwtal & $\begin{array}{l}\text { Leaves and } \\
\text { shoot }\end{array}$ & Herb & $\begin{array}{l}\text { Leaves used as vegetable (commonly called saag) and as fodder } \\
\text { for livestock. }\end{array}$ \\
\hline \multicolumn{5}{|l|}{ Juglandaceae } \\
\hline Juglans regia L. & Ghuz & Whole plant & Tree & $\begin{array}{l}\text { The wood is used in quality furniture, leaves, and roots used for } \\
\text { teeth cleaning and as lipstick (locally called Dandasa); fruit oils } \\
\text { used as brain tonic. }\end{array}$ \\
\hline \multicolumn{5}{|l|}{ Lamiaceae } \\
\hline Ajuga bracteosa Wall ex. Benth & Khwaga / Bootei & Shoot and root & Herb & $\begin{array}{l}\text { Shoot and root against constipation, cough, as blood purifier, } \\
\text { cooling agent. }\end{array}$ \\
\hline Ajuga parviflora Benth. & Khwaga / Bootei & Leaves & Herb & Leaves are considered carminative, anti-jaundice \\
\hline
\end{tabular}




\begin{tabular}{|c|c|c|c|c|}
\hline Mentha arvensis L. & Podina & Leaves & Herb & $\begin{array}{l}\text { As a digestive and stimulating agent, salad, criminative, } \\
\text { expectorant, gastric problems. }\end{array}$ \\
\hline Mentha longifolia (L.) Huds. & Enaly / wenaly & Leaves & Herb & Leaves are stimulants, astringent, against stomach-ache. \\
\hline Ocimum basilicum $\mathrm{L}$. & Kashmalyi & $\begin{array}{l}\text { Seeds and } \\
\text { leaves }\end{array}$ & Herb & $\begin{array}{l}\text { As an ornamental plant commonly in the home as a fragrance } \\
\text { agent, leaves are mostly used against cough, seeds (in cold } \\
\text { water) used against vomiting. }\end{array}$ \\
\hline Origanum vulgare $\mathrm{L}$. & Jangali & Shoot & Herb & Diuretic and flavoring agent. \\
\hline Otostegia limbata (Ben th.) Boiss. & Zerakay & Leaves & Herb & $\begin{array}{l}\text { Leaves are effective against gum problems and other small } \\
\text { wounds. }\end{array}$ \\
\hline Salvia plebeian $\mathrm{R}$. Br. & Sheenkay & Shoot & Herb & Anti-diarrheal, against urinary tract irritation. \\
\hline \multicolumn{5}{|l|}{ Moraceae } \\
\hline Ficus carica L. & Inzaar & $\begin{array}{l}\text { Fruits and } \\
\text { Latex }\end{array}$ & Tree & $\begin{array}{l}\text { Fruits for stomach problems, constipation, urinary and bladder } \\
\text { infection, latex used to remove warts and scab from the body. }\end{array}$ \\
\hline Morus alba L. & Speen tut & $\begin{array}{l}\text { Fruits and } \\
\text { stem }\end{array}$ & Tree & $\begin{array}{l}\text { Wood used for furniture and as fuel, fruits against joints pain } \\
\text { (rheumatism) }\end{array}$ \\
\hline Morus nigra L. & Khary tut / Toor tut & Stem and fruit & Tree & $\begin{array}{l}\text { The wood is used in furniture and fuel, fruit used as a tonic and } \\
\text { against cough (in dried form) }\end{array}$ \\
\hline \multicolumn{5}{|l|}{ Morchellaceae } \\
\hline Morchella esculenta Fr. & Gosay & Whole & Mushroom & Used as a high energy source \\
\hline \multicolumn{5}{|l|}{ Nyctaginaceae } \\
\hline Boerhavia diffusa L. & Abi gul & Whole plant & Herb & Diuretic, wound healing \\
\hline Mirabilis jalapa L. & Mazigar gully & $\begin{array}{l}\text { Seeds and, } \\
\text { leaves }\end{array}$ & Herb & Seeds and leaves are used for wound healing, urinary disorders. \\
\hline \multicolumn{5}{|l|}{ Oleaceae } \\
\hline $\begin{array}{l}\text { Olea europaea subsp. cuspidata (Wall. \& G. } \\
\text { Don) Cif. }\end{array}$ & Khoonah/ zaitoon & $\begin{array}{l}\text { Leaves and } \\
\text { oils }\end{array}$ & Tree & $\begin{array}{l}\text { Leaves are used to treat throat problems and diabetes, oils } \\
\text { antibacterial }\end{array}$ \\
\hline \multicolumn{5}{|l|}{ Papaveraceae } \\
\hline Papaver pavoninum schrenk. & Sur gulay & Fruit, seeds & Herb & For chest trouble, cough, diarrhea. \\
\hline Papaver somniferum L. & Apium & Seeds, latex & Herb & $\begin{array}{l}\text { Latex and seeds are used against diebetes and as a stimulant, } \\
\text { for respiratory problem. }\end{array}$ \\
\hline \multicolumn{5}{|l|}{ Pinaceae } \\
\hline Pinus roxburghii Sarg & Nakhtar & $\begin{array}{l}\text { Gum, stem, } \\
\text { fruits }\end{array}$ & Tree & Use against various skin problems, to remove pus from wounds \\
\hline Pinus wallichiana A. B. Jacks & Nakhtar & $\begin{array}{l}\text { Gums, stem, } \\
\text { fruits }\end{array}$ & Tree & $\begin{array}{l}\text { Stems mostly used for furniture, dried leaves as fuel } \\
\text { (domestically), fruits as body and brain tonic. }\end{array}$ \\
\hline \multicolumn{5}{|l|}{ Poaceae } \\
\hline Aristida cyanatha & Waakha & Whole plant & Grass & Fodder for cattle. \\
\hline Avena sativa $\mathrm{L}$. & Jawdar/jamdar & Whole plants & Herb & Seeds used forstomach problems, fodder. \\
\hline Cynodon dactylon (L.) Pers & Kabll & Whole plant & Herb & odder, ornamental, anti-constipation. \\
\hline \multicolumn{5}{|l|}{ Polygonaceae } \\
\hline Rumex abyssinicus Jacq. & Taaroki & Leaves & Herb & Diuretic, carminative. \\
\hline
\end{tabular}




\begin{tabular}{|c|c|c|c|c|}
\hline \multicolumn{5}{|l|}{ Rhamnaceae } \\
\hline Zizyphus mauritiana Lam. & Markhanai/ nagara & $\begin{array}{l}\text { Fruits and } \\
\text { leaves }\end{array}$ & Tree & Leaves are widely used against diabetes, fruits are emollient. \\
\hline \multicolumn{5}{|l|}{ Rosaceae } \\
\hline Duchesnea indica (Andrews.) Focke. & Janati mewa & Fruits & Herb & Fruits are used for eye infection and as a tonic. \\
\hline Pyrus communis L. & Nashpati & Fruits & Tree & Fruit is laxative and spasmodic. \\
\hline Rubus ellipticus Sm. & Ziyra karwara & Fruits & Shrub & Fruits are used against vomiting and diarrhea. \\
\hline Rubus fruticosus $\mathrm{L}$. & Karwara & Whole plant & Shrub & Plants are used for living fences, fruits edible. \\
\hline \multicolumn{5}{|l|}{ Salicaceae } \\
\hline Populus nigra L. & Supidar & Stem & Tree & Stem used for furniture, matches, fuel. \\
\hline Salix alba L. & Wala & $\begin{array}{l}\text { Leaves and } \\
\text { bark }\end{array}$ & Tree & Bark used to reduce sharp fever, leaves analgesic. \\
\hline \multicolumn{5}{|l|}{ Sapindaceae } \\
\hline Dodonaea viscosa Jacq. & Ghwarsky & $\begin{array}{l}\text { Seeds, leaves, } \\
\text { and stem. }\end{array}$ & Shrub & $\begin{array}{l}\text { Leaves and seeds are used for wound healing (to remove pus), } \\
\text { the stem is used as fuel. }\end{array}$ \\
\hline \multicolumn{5}{|r|}{ (1) } \\
\hline Monotheca buxifolia (A. DC.) T.D. Penn & Gwargwara & $\begin{array}{l}\text { Fruits and } \\
\text { stem }\end{array}$ & Shrub & Fruits are edible, wood is used for fuel. \\
\hline \multicolumn{5}{|l|}{ Simaroubaceae } \\
\hline Ailanthus altissima (Mill.) Swingle & Farmai shandai & $\begin{array}{l}\text { Stem and } \\
\text { leaves }\end{array}$ & Tree & Leaves are used as fodder, stem as fuelwood. \\
\hline \multicolumn{5}{|l|}{ Solanaceae } \\
\hline Datura innoxia Mill. & Datura & $\begin{array}{l}\text { Leaves and } \\
\text { fruits }\end{array}$ & Shrub & $\begin{array}{l}\text { Leaf as bandage to control bleeding, boiled seeds used against } \\
\text { asthma. }\end{array}$ \\
\hline Solanum nigrum $\mathrm{L}$. & Karmachu & Fruits & Herb & Fruits are used against fever, joint pain. \\
\hline Solanum surattense Burm. f. & Anda botay & Seed & Herb & Seeds are used against headache, antidiabetic. \\
\hline Withania somnifera (L.) Dunal & $\begin{array}{l}\text { Khamazoory/ } \\
\text { Ashwagandha }\end{array}$ & Seed & Shrub & Seeds for wounds, to regulate body temperature. \\
\hline \multicolumn{5}{|l|}{ Zygophyllacae } \\
\hline Tribulus terrestris L. & Markondayai & Seed & Herb & Seeds for urinary infections, as tonic. \\
\hline
\end{tabular}




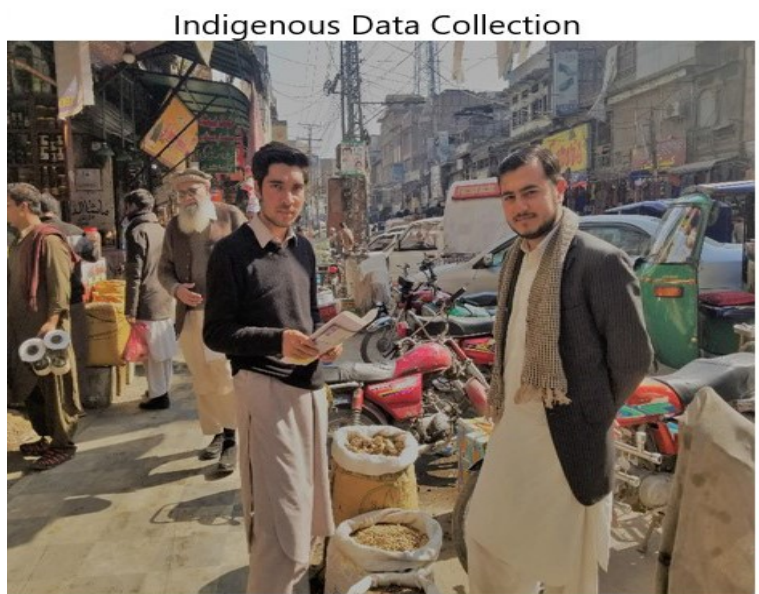

Papaver somniferum L. (Poppies)

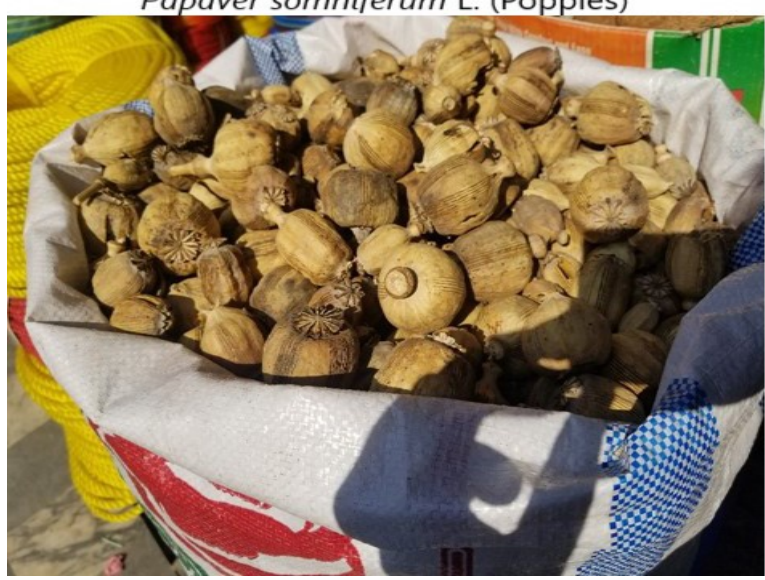

Figure 2. Data collection

Table 2. Ethnobotanical diversity

\begin{tabular}{|l|l|}
\hline Family Name & No. of species \\
\hline Adiantaceae & 1 \\
\hline Agaricaceae & 1 \\
\hline Alismataceae & 1 \\
\hline Amaranthaceae & 6 \\
\hline Amaryllidaceae & 2 \\
\hline Apiaceae & 3 \\
\hline Apocynaceae & 2 \\
\hline Asphodelaceae & 1 \\
\hline Asteraceae & 9 \\
\hline Berberidaceae & 1 \\
\hline Brassicaceae & 5 \\
\hline Cannabaceae & 1 \\
\hline Cucurbitaceae & 6 \\
\hline Cupressaceae & 1 \\
\hline Ebenaceae & 1 \\
\hline Equisetaceae & 1 \\
\hline Euphorbiaceae & 4 \\
\hline Fabaceae & 5 \\
\hline Juglandaceae & 1 \\
\hline Lamiaceae & 8 \\
\hline Moraceae & 3 \\
\hline Morchellaceae & 1 \\
\hline
\end{tabular}

Medicinal Plants Fruits, Stem, Seeds, and leaves

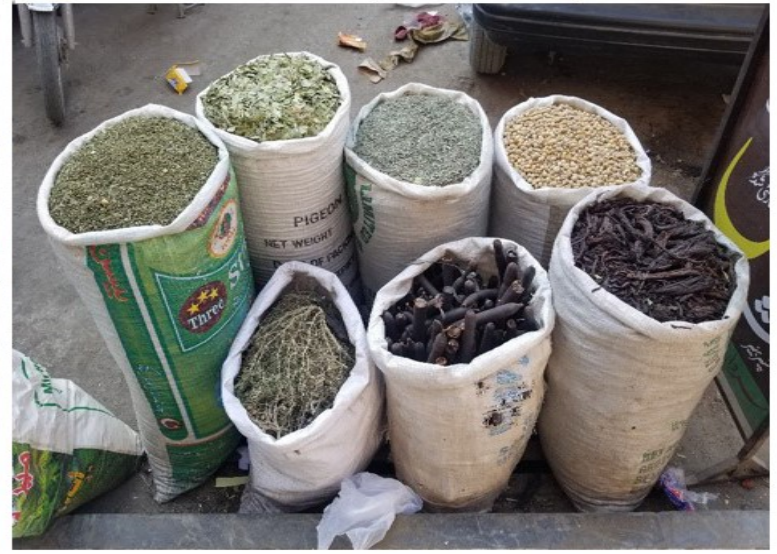

Data Confirmation from Hakeem

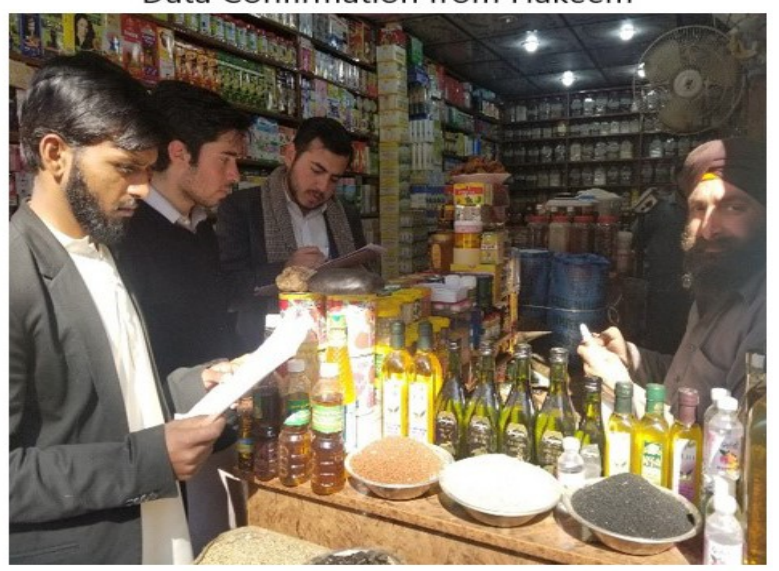

\begin{tabular}{|l|l|}
\hline Nyctaginaceae & 2 \\
\hline Oleaceae & 1 \\
\hline Papaveraceae & 2 \\
\hline Pinaceae & 2 \\
\hline Poaceae & 3 \\
\hline Polygonaceae & 1 \\
\hline Rhamnaceae & 1 \\
\hline Rosaceae & 4 \\
\hline Salicaceae & 2 \\
\hline Sapindaceae & 1 \\
\hline Sapotaceae & 1 \\
\hline Simaroubaceae & 1 \\
\hline Solanaceae & 4 \\
\hline Zygophyllacae & 1 \\
\hline
\end{tabular}

\section{Form and mode of utilization}

Out of the 92 plant species, 43 plants were medicinal and were used against multiple ailments, while 13 plant species were used as fuel and timber, 8 species were used to construct shelter, 23 plants were used as a source of food and energy, and 13 plants were used as fodder for livestock as mentioned in Figure 6. Out of the 43 recorded medicinal species, 6 were used against respiratory disorders 3 plants were used anti-constipation. Figure 5. 


\section{- Herbs \\ - Shrubs \\ - Trees \\ - Fungi}

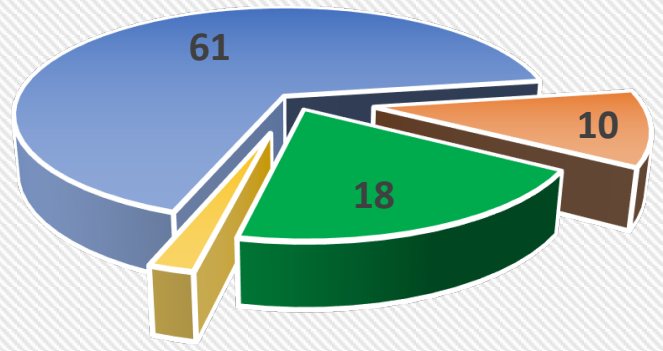

2

Figure 3. Life forms of plants used.

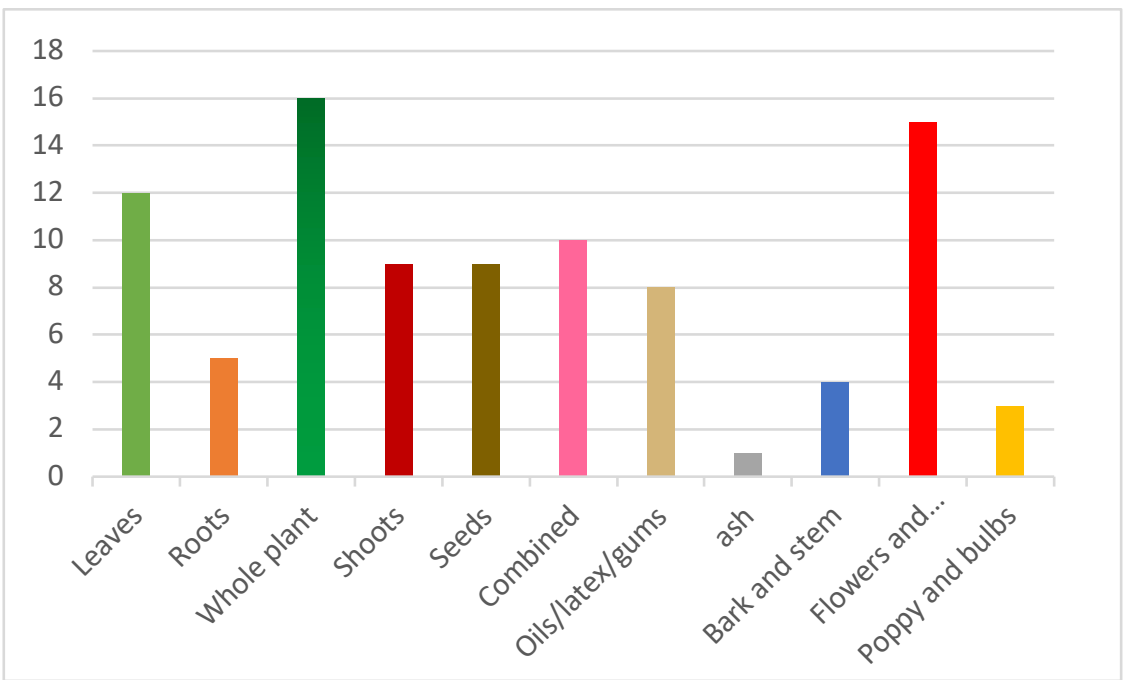

Figure 4. Plant parts used.

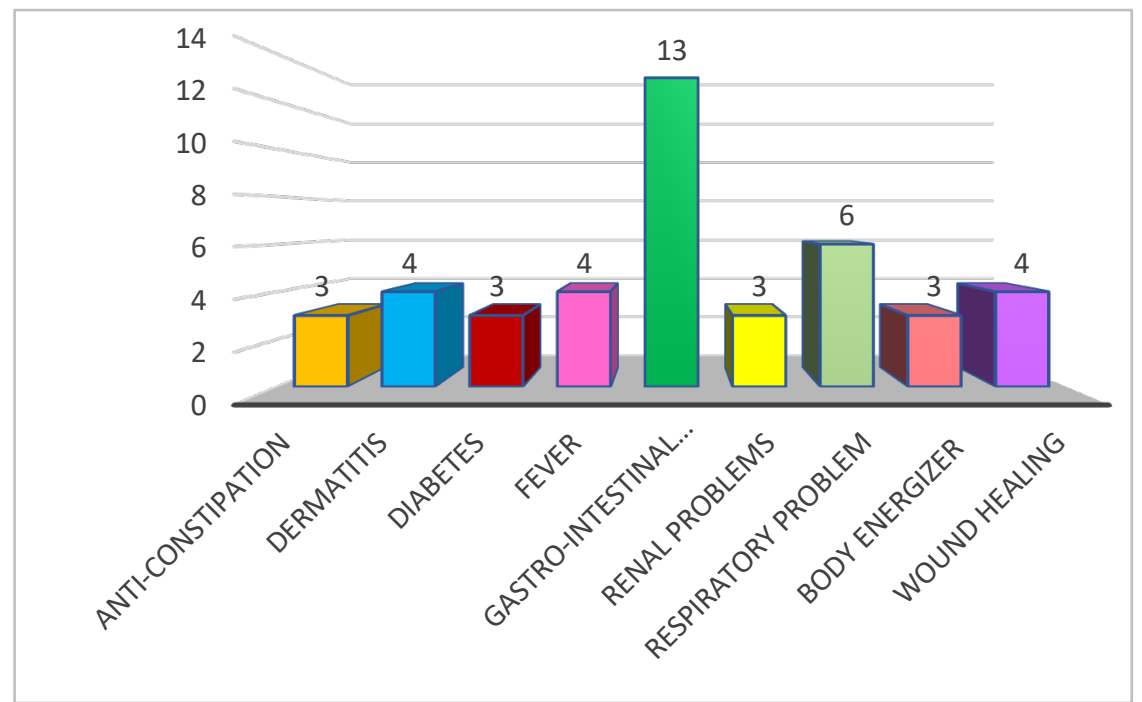

Figure 5. Disease category 


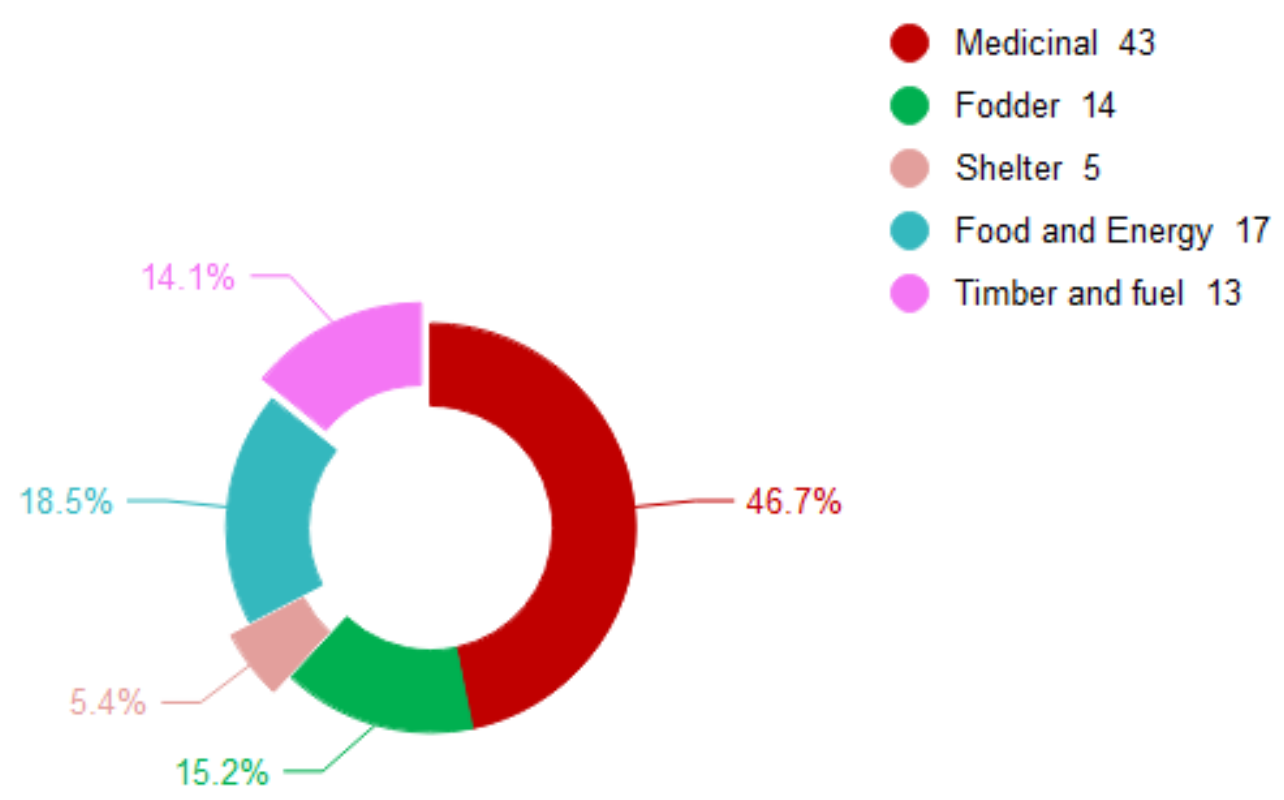

Figure 6. Form and mode of utilization

\section{Discussion}

Our study indicated that the residents of the research area still uses a wide variety of plants for their daily needs such as cash income, for fuel, medicine, fodder, shelter, etc. Asteraceae and Lamiaceae were the most dominant families in the research area. Acacia nilotica Withania somnifera, and Berberis lycium were the most cited plants by the residents. According to available literature Berberis lycium, Cannabis sativa, Ammi visnaga and Dodonaea viscosa showed highly medicinal properties (Ahmad et al. 2013), although also being heavily used as fuel and fodder. Some species like Papaver somniferum, Cannabis sativa, Acacia arabica, were found to be very effective against respiratory problems (Jindal et al. 2012). Equisitum arvense and Ammi visnaga have been shown to control sharp fever and wounds, similar to our results (Lorenzen et al. 2011). Rapee et al. (2005) also found Taraxacum officinale as a stimulating agent and energizer. Rani and Mohan (2009) documented that Dodonaea viscosa leaves and seeds were used for wound healing. Mentha arvensis leaves are widely used as digestive and stimulating agents, effective against gastric problems, expectorant, and have criminative properties, and our data matched with (Biswas et al. 2014).

Few members of Family Apiaceae like Coriandrum sativum and Foeniculum vulgare were considered strong digestive and kitchen agents, used as condiments and a flavoring mediator similar to (Asowata-Ayodele 2015). Some medicinal plants are under serve threat, due to over-harvesting, including Dalbergia sisso, Pinus roxburghii and Acacia modesta, which were mainly targeted by the timber mafia. The most common market is the furniture industry. The same species were also recklessly cut to construct shelters and homes. Some bold and serious steps are needed to protect the local biodiversity and ecosystems from such practices. We hope our study will be a baseline to create awareness in local bodies and forest departments to preserve the unique flora and fauna existing in the area and exercise some permanent laws to stop the over-exploitation of natural resources. We hope to encourage the residents to protect the natural value of the area. This would also facilitate national and international tourism, which could boost the economy and contribute to the health sector.

\section{Conclusion}

It is concluded from this survey that the area lacks modern facilities, and the majority of the inhabitants are poor and have no permanent and stable source of income. Due to these reasons the residents mostly depend on plants for medicinal use, and other basic needs such as fodder, timber, fuel, and construction. The medicinal flora is under severe 
threat due to over harvesting because of its high cash value, as well as the impacts of population explosion. Some plants like Dalbergia spp., Pinus spp. and Acacia spp. are multipurpose plants that need serious management and conservation attention. The medicinal flora of the area and the vegetation in general are under the stress of overuse. Immediate conservation programs, reforestation, forest guard management, solid laws, and awareness programs for the local population are urgently needed.

\section{Declarations}

List of abbreviations: N/A

Ethics approval and consent to participate: All participants provided oral prior informed consent

Consent for publication: N/A

Funding: Self-support project. This project got not any fund or sponsorship.

Authors' contributions: Murad Muhammad and Rainer W. Bussmann wrote and reviewed the original manuscript as well as the final manuscript; Lal Badshah and Abdul Basit conceived and designed the study; Adnan Ali Shah, Abdullah, and Muhammad Ali Shah. contributed during field visits and data collection.

Competing interest: The authors have no competing interests.

\section{Acknowledgments}

All the authors are grateful to the residents, herbalists, and plant traders for sharing their authentic and experienced knowledge, ideas, suggestions, and recommendations as to be preserved for future references.

\section{Literature cited}

Abbas A, Khan SM, Alam J, Khan SW, Abbasi AM. 2017. Medicinal plants used by inhabitants of the Shigar Valley, Baltistan region of Karakorum rangePakistan. Journal of Ethnobiology and Ethnomedicine 13(1):1-15.

Abbas W, Hussain W, Hussain W, Badshah L, Hussain K, Pieroni A. 2020. Traditional wild vegetables gathered by four religious groups in Kurram District, Khyber Pakhtunkhwa, North-West Pakistan, Genetic Resources and Crop Evolution 67(6):1521-1536.

Ahmad A, Husain A, Mujeeb M, Khan SA, Najmi AK, Siddique NA, Anwar F. 2013. A review on therapeutic potential of Nigella sativa: A miracle herb. Asian Pacific Journal of Tropical Biomedicine 3(5):337-352.

Aloi G. 2018. Why Look at Plants?: The Botanical Emergence in Contemporary Art. Brill, Leiden.
Asowata-Ayodele AM 2015. Pharmacological studies of Foeniculum vulgare (Mill.) and Lippia javanica (Burm. f.) Spreng. used as spices in Nkonkobe Municipality of the Eastern Cape province, South Africa. University of Fort Hare. http://hdl.handle.net/20.500.11837/241

Balázs VL, Filep R, Ambrus T, Kocsis M, Farkas Á, Stranczinger S, Papp N. 202. Ethnobotanical, historical and histological evaluation of Helleborus $L$. genetic resources used in veterinary and human ethnomedicine. Genetic Resources and Crop Evolution, 67(3):781-797.

Biswas A, Mandal A, Sharma S, Mohanty W. 2014. Delineation of subsurface structures using selfpotential, gravity, and resistivity surveys from South Purulia Shear Zone, India: Implication to uranium mineralization. Interpretation 2(2):T103-T110.

Chauhan K. 2020. Role of Ethnobotany on Indian Society. Journal of Arts, Culture, Philosophy, Religion, Language and Literature 4(2):109-111.

Chetry LB, Basar K, Taye K, Taka T, Tsering J, Wangpan T, Bharali MK. 2018. Medicinal Plants used against gastrointestinal disorders among the Adi Tribe of Eastern Himalaya. NeBIO 9(1):93-101.

Cock I, Selesho M, van Vuuren S. 2018. A review of the traditional use of southern African medicinal plants for the treatment of selected parasite infections affecting humans, Journal of Ethnopharmacology 220:250-264.

Dapar MLG, Alejandro GJD, Meve U, LiedeSchumann S. 2020. Quantitative ethnopharmacological documentation and molecular confirmation of medicinal plants used by the Manobo tribe of Agusan del Sur, Philippines, Journal of Ethnobiology and Ethnomedicine 16 (1):1-60.

Elachouri M, Kharchoufa L, Fakchich J, Lorigooini Z, Subhasis P, Subhash M. 2021. Ancestral phytotherapeutic practices in Morocco: regards on history, current state, regulatory and safety of commonly used herbal medicine, Arabian Journal of Chemical and Environmental Research 8(1): 133149.

Elahi K, ur Rahman A 2021. An Analysis of Women Voters as a Factor in JIP's Electoral Performance in Upper Dir \& Lower Dir in General Elections 2018. The Dialogue 16(1):22-38.

Hubberten HW, Bolshiyanov DY, Grigoriev MN, Grosse G, Morgenstern A, Pfeiffer EM, Schirrmeister L. 2018. 20 Years of Terrestrial Research in the Siberian Arctic, The History of the Lena Expeditions. Alfred Wegener Institute Helmholtz Centre for Polar and Marine Research.

Jan HA, Jan S, Bussmann RW, Wali S, Sisto F, Ahmad L. 2020. Complementary and alternative medicine research, prospects and limitations in 
Pakistan: A literature review. Acta Ecologica Sinica 40(6):451-463.

Jindal SK, Aggarwal AN, Gupta D, Agarwal R, Kumar R, Kaur T, Shah B. 2012. Indian study on epidemiology of asthma, respiratory symptoms and chronic bronchitis in adults (INSEARCH). The International Journal of Tuberculosis and Lung Disease 16(9):1270-1277.

Latimer A. 2020. Encoded knowledge in oral traditions: Skwxwú7mesh transformer sites and their relationship with landscape perception and use (Doctoral dissertation, Environment: Department of Archaeology, Simon Frazer University).

Lorenzen ED, Nogués-Bravo D, Orlando L, Weinstock J, Binladen J, Marske KA, Willerslev E. 2011. Species-specific responses of Late Quaternary megafauna to climate and humans. Nature 479(7373):359-364.

Majid A, Ahmad H, Saqib Z, Rahman IU, Khan U, Alam J, Ali N. 2019. Exploring threatened traditional knowledge; ethnomedicinal studies of rare endemic flora from Lesser Himalayan region of Pakistan. Revista Brasileira de Farmacognosia 29(6):785-792.

Pandey AK, Tripathi Y. 2017. Ethnobotany and its relevance in contemporary research, Journal of Medicinal Plant Studies 5(3):123-129.

Punchay K, Inta A, Tiansawat P, Balslev H, Wangpakapattanawong P. 2020. Traditional knowledge of wild food plants of Thai Karen and Lawa (Thailand). Genetic Resources and Crop Evolution 67(5):1277-129.
Rani MS, Mohan RSPK. 2009. Dodonaea viscosa Linn. - an overview. Asian Journal of Pharmaceutical Research and Health Care 1(1).

Rapee RM, Kennedy S, Ingram M, Edwards S, Sweeney L. 2005. Prevention and early intervention of anxiety disorders in inhibited preschool children, Journal of Consulting and Clinical Psychology 73(3):488.

Santos FO, Cerqueira APM, Branco A, Batatinha MJM, Botura MB. 2019. Anthelmintic activity of plants against gastrointestinal nematodes of goats: a review. Parasitology 146(10):1233-1246.

Singh A, Pal K. 2017. Ethnobotanical and antibacterial study of some selected medicinal plants in Haryana, International Journal of Research in Medical and Basic Sciences 03(09).

Sutrisno IH, Akob B, Navia ZI, Nuraini N, Suwardi AB. 2020. Documentation of ritual plants used among the Aceh tribe in Peureulak, East Aceh District, Indonesia. Biodiversitas - Journal of Biological Diversity 21(11).

Umair M, Altaf M, Abbasi AM. 2017. An ethnobotanical survey of indigenous medicinal plants in Hafizabad district, Punjab-Pakistan, PloS one 12(6):e0177912.

Witt A, Beale T, van Wilgen BW. 2018. An assessment of the distribution and potential ecological impacts of invasive alien plant species in eastern Africa, Transactions of the Royal Society of South Africa 73(3):217-236. 


\section{Appendix}

\section{Structured Questionnaire}

\section{Preliminary questions}

- Respondent name:

- Respondent age:.

Respondents total duration or residence around study area:...years

\section{Plant Attributes}

Local name:

Plant form (tick as appropriate. Filled in by interviewer from observation):

Tree..........Shrub............ Herb.........Forb............Sedge.........Grass.......

Climber.............. Other(specify) $\ldots \ldots \ldots \ldots \ldots \ldots \ldots$

Plant origin (tick as appropriate):

Indigenous...............Exotic.

Common (English) name: to be filled in by interviewer

Family: (to be filled in by interviewer):

Scientific name (to be filled in by interviewer):.

\section{Collection site}

In relation to forest (Tick as appropriate)

Inside.

Outside):.

Forest block name:

\section{Symptoms or condition cured (List)}

1.

2.

3.

4.

5.

\section{Collection site description}

Part(s) used or from which medicine is extracted (tick as appropriate)

Leaves

Bark ....

Roots

Fruits

Flowers

Other (specify)

\section{General preparation method}


Method of administering medication.

Patient age group (tick as appropriate)

Elderly

Adults.

Youth.

Children.

Infants.

All age groups

Patient gender (tick as appropriate)

Male

Female.

Both genders 